\title{
Fourier series of functions associated with higher-order Bernoulli polynomials
}

\author{
Taekyun Kimª,b, Dae San Kimc, Dmitry V. Dolgy ${ }^{\mathrm{d}}$, Jin-Woo Park ${ }^{\mathrm{e}, *}$ \\ ${ }^{a}$ Department of Mathematics, College of Science, Tianjin Polytechnic University, Tianjin, 300160, China. \\ ${ }^{b}$ Department of Mathematics, College of Natural Science, Kwangwoon University, Seoul 139-701, Republic of Korea. \\ ${ }^{c}$ Department of Mathematics, Sogang University, Seoul 121-742, Republic of Korea. \\ ${ }^{d}$ Hrimwon, Kwangwoon University, Seoul 139-701, Republic of Korea. \\ ${ }^{e}$ Department of Mathematics Education, Daegu University, Gyeongsan-si, Gyeongsangbuk-do, 712-714, Republic of Korea. \\ Communicated by Sh. Wu
}

\begin{abstract}
In this paper, we consider three types of functions associated with higher-order Bernoulli polynomials and derive their Fourier series expansions. Further, we express each of them in term of Bernoulli functions. (c)2017 All rights reserved.
\end{abstract}

Keywords: Fourier series, higher-order Bernoulli polynomials, Bernoulli functions.

2010 MSC: 11B68, 11B83, 42A16.

\section{Introduction}

For each positive integer $r$, Bernoulli polynomials $B_{m}^{(r)}(x)$ of order $r$ are given by the generating function

$$
\left(\frac{t}{e^{t}-1}\right)^{r} e^{x t}=\sum_{m=0}^{\infty} B_{m}^{(r)}(x) \frac{t^{m}}{m !}, \quad(\text { see }[2,6,8,10,12,14,17]) .
$$

When $x=0, B_{m}^{(r)}=B_{m}^{(r)}(0)$ are called Bernoulli numbers of order $r$. For $r=1, B_{m}(x)=B_{m}^{(1)}(x)$ and $\mathrm{B}_{\mathrm{m}}=\mathrm{B}_{\mathrm{m}}^{(1)}$ are called Bernoulli polynomials and Bernoulli numbers, respectively.

From (1.1), we see that

$$
\begin{aligned}
\frac{d}{d x} B_{m}^{(r)}(x) & =m B_{m-1}^{(r)}(x), \quad(m \geqslant 0), \\
B_{m}^{(r)}(x+1) & =B_{m}^{(r)}(x)+m B_{m-1}^{(r-1)}(x), \quad(m \geqslant 0) .
\end{aligned}
$$

\footnotetext{
${ }^{*}$ Corresponding author

Email addresses: tkkim@kw.ac.kr (Taekyun Kim), dskim@sogang.ac.kr (Dae San Kim), dvdolgy@gmail.com (Dmitry V. Dolgy), a0417001@knu.ac.kr (Jin-Woo Park) 
In turn, these yield

$$
\begin{gathered}
B_{m}^{(r)}(1)=B_{m}^{(r)}+m B_{m-1}^{(r-1)}, \quad(m \geqslant 0), \\
\int_{0}^{1} B_{m}^{(r)}(x) d x=B_{m}^{(r-1)}, \quad(m \geqslant 0) .
\end{gathered}
$$

For any real number $x$, we let

$$
\langle x\rangle=x-\lfloor x\rfloor \in[0,1),
$$

denote the fractional part of $x$.

For later use, we recall the following facts about Bernoulli functions $B_{m}(\langle x\rangle)$ :

(a) for $m \geqslant 2$,

$$
B_{m}(x)=-m ! \sum_{\substack{n=-\infty \\ n \neq 0}}^{\infty} \frac{e^{2 \pi i n x}}{(2 \pi i n)^{m}}
$$

(b) for $m=1$,

$$
-\sum_{\substack{n=-\infty \\
n \neq 0}}^{\infty} \frac{e^{2 \pi i n x}}{2 \pi i n}=\left\{\begin{array}{cc}
B_{1}(\langle x\rangle), & \text { for } x \notin \mathbb{Z}, \\
0, & \text { for } x \in \mathbb{Z} .
\end{array}\right.
$$

Here we will consider the following three types of functions $\alpha_{m}(x), \beta_{m}(x)$, and $\gamma_{m}(x)$ associated with higher-order Bernoulli polynomials. We will derive their Fourier series expansions and further express them in term of Bernoulli functions.
(a) $\alpha_{m}(\langle x\rangle)=\sum_{k=0}^{m} B_{k}^{(r)}(\langle x\rangle)\langle x\rangle^{m-k}, \quad(m \geqslant 1)$;
(b) $\beta_{m}(\langle x\rangle)=\sum_{k=0}^{m} \frac{1}{k !(m-k) !} B_{k}^{(r)}(\langle x\rangle)\langle x\rangle^{m-k}, \quad(m \geqslant 1)$;
(c) $\gamma_{m}(\langle x\rangle)=\sum_{k=1}^{m-1} \frac{1}{k(m-k)} B_{k}^{(r)}(\langle x\rangle)\langle x\rangle^{m-k}, \quad(m \geqslant 2)$.

For elementary facts about Fourier analysis, the reader may refer to any book (for example, see $[1,15,18]$ ).

As to $\gamma_{m}(\langle x\rangle)$, we note that the polynomial identity (1.2) follows immediately from Theorems 4.1 and 4.2 which is in turn derived from the Fourier series expansion of $\gamma_{m}(\langle x\rangle)$.

$$
\begin{aligned}
& \sum_{k=1}^{m-1} \frac{1}{k(m-k)} B_{k}^{(r)}(x) x^{m-k} \\
& \quad=\frac{1}{m} \sum_{s=0}^{m}\left(\begin{array}{c}
m \\
s
\end{array}\right)\left(\Lambda_{m-s+1}+\left(H_{m-1}-H_{m-s}\right)\left(\frac{1}{m-s+1}+B_{m-s}^{(r-1)}\right)\right) B_{s}(x)
\end{aligned}
$$

where $\Lambda_{l}=\sum_{k=1}^{l-1} \frac{1}{k(l-k)}\left(B_{k}^{(r)}+k B_{k-1}^{(r-1)}\right)$, for $l \geqslant 2$, with $\Lambda_{1}=0$, and $H_{m}=\sum_{j=1}^{m} \frac{1}{j}$ are the harmonic numbers.

The obvious polynomial identities can be derived also for $\alpha_{m}(\langle x\rangle)$ and $\beta_{m}(\langle x\rangle)$ from Theorems 2.1 and 2.2, and Theorems 3.1 and 3.2, respectively. It is remarkable that from the Fourier series expansion of the function $\sum_{k=1}^{m-1} \frac{1}{k(m-k)} B_{k}(\langle x\rangle) B_{m-k}(\langle x\rangle)$ we can derive the Faber-Pandharipande-Zagier identity (see $[4,7,9])$ and the Miki's identity (see $[3,5,7,9,16])$. For recent related works, we refer the reader to $[11,13]$.

\section{Fourier series of functions of the first type}

Let

$$
\alpha_{m}(x)=\sum_{k=0}^{m} B_{k}^{(r)}(x) x^{m-k}, \quad(m \geqslant 1) .
$$


Then we will consider the function

$$
\alpha_{m}(\langle x\rangle)=\sum_{k=0}^{m} B_{k}^{(r)}(\langle x\rangle)\langle x\rangle^{m-k},
$$

defined on $\mathbb{R}$ which is periodic with period 1 .

The Fourier series of $\alpha_{m}(\langle x\rangle)$ is

$$
\sum_{n=-\infty}^{\infty} A_{n}^{(m)} e^{2 \pi i n x}
$$

where

$$
\begin{aligned}
A_{n}^{(m)} & =\int_{0}^{1} \alpha_{m}(\langle x\rangle) e^{-2 \pi i n x} d x \\
& =\int_{0}^{1} \alpha_{m}(x) e^{-2 \pi i n x} d x .
\end{aligned}
$$

To proceed further, we need to note the following.

$$
\begin{aligned}
\alpha_{m}^{\prime}(x) & =\sum_{k=0}^{m}\left\{k B_{k-1}^{(r)}(x) x^{m-k}+(m-k) B_{k}^{(r)}(x) x^{m-k-1}\right\} \\
& =\sum_{k=1}^{m} k B_{k-1}^{(r)}(x) x^{m-k}+\sum_{k=0}^{m-1}(m-k) B_{k}^{(r)}(x) x^{m-k-1} \\
& =\sum_{k=0}^{m-1}(k+1) B_{k}^{(r)}(x) x^{m-k-1}+\sum_{k=0}^{m-1}(m-k) B_{k}^{(r)}(x) x^{m-k-1} \\
& =(m+1) \sum_{k=0}^{m-1} B_{k}^{(r)}(x) x^{m-1-k} \\
& =(m+1) \alpha_{m-1}(x) .
\end{aligned}
$$

From this, we obtain

$$
\left(\frac{\alpha_{m+1}(x)}{m+2}\right)^{\prime}=\alpha_{m}(x)
$$

and

$$
\int_{0}^{1} \alpha_{m}(x) d x=\frac{1}{m+2}\left(\alpha_{m+1}(1)-\alpha_{m+1}(0)\right) .
$$

For $m \geqslant 1$, we put

$$
\begin{aligned}
\Delta_{m} & =\alpha_{m}(1)-\alpha_{m}(0) \\
& =\sum_{k=0}^{m}\left(B_{k}^{(r)}(1)-B_{k}^{(r)} \delta_{m, k}\right) \\
& =\sum_{k=0}^{m}\left(B_{k}^{(r)}+k B_{k-1}^{(r-1)}-B_{k}^{(r)} \delta_{m, k}\right) \\
& =\sum_{k=0}^{m} B_{k}^{(r)}+\sum_{k=1}^{m} k B_{k-1}^{(r-1)}-B_{m}^{(r)} \\
& =\sum_{k=0}^{m-1}\left(B_{k}^{(r)}+(k+1) B_{k}^{(r-1)}\right) .
\end{aligned}
$$


We now observe that

$$
\alpha_{\mathrm{m}}(0)=\alpha_{\mathrm{m}}(1) \Longleftrightarrow \Delta_{\mathrm{m}}=0,
$$

and

$$
\begin{aligned}
\int_{0}^{1} \alpha_{m}(x) d x & =\frac{1}{m+2} \Delta_{m+1} \\
& =\frac{1}{m+2} \sum_{k=0}^{m}\left(B_{k}^{(r)}+(k+1) B_{k}^{(r-1)}\right) .
\end{aligned}
$$

We are now ready to determine the Fourier coefficients $A_{n}^{(m)}$.

Case $1: n \neq 0$.

$$
\begin{aligned}
A_{n}^{(m)} & =\int_{0}^{1} \alpha_{m}(x) e^{-2 \pi i n x} d x \\
& =-\frac{1}{2 \pi i n}\left[\alpha_{m}(x) e^{-2 \pi i n x}\right]_{0}^{1}+\frac{1}{2 \pi i n} \int_{0}^{1} \alpha_{m}^{\prime}(x) e^{-2 \pi i n x} d x \\
& =-\frac{1}{2 \pi i n}\left(\alpha_{m}(1)-\alpha_{m}(0)\right)+\frac{m+1}{2 \pi i n} \int_{0}^{1} \alpha_{m-1}(x) e^{-2 \pi i n x} d x \\
& =\frac{m+1}{2 \pi i n} A_{n}^{(m-1)}-\frac{1}{2 \pi i n} \Delta_{m}
\end{aligned}
$$

from which by induction on $m$ we can show that

$$
A_{n}^{(m)}=-\frac{1}{m+2} \sum_{j=1}^{m} \frac{(m+2)_{j}}{(2 \pi i n)^{j}} \Delta_{m-j+1} .
$$

Case $2: n=0$.

$$
A_{0}^{(m)}=\int_{0}^{1} \alpha_{m}(x) d x=\frac{1}{m+2} \Delta_{m+1} .
$$

$\alpha_{m}(\langle x\rangle),(m \geqslant 1)$ is piecewise $C^{\infty}$. Moreover, $\alpha_{m}(\langle x\rangle)$ is continuous for those positive integers $m$ with $\Delta_{\mathrm{m}}=0$, and discontinuous with jump discontinuities at integers for those positive integers with $\Delta_{\mathrm{m}} \neq 0$.

Assume first that $\mathrm{m}$ is a positive integer with $\Delta_{m}=0$. Then $\alpha_{m}(0)=\alpha_{m}(1)$. Hence $\alpha_{m}(\langle x\rangle)$ is piecewise $C^{\infty}$, and continuous. Thus the Fourier series of $\alpha_{m}(\langle x\rangle)$ converges uniformly to $\alpha_{m}(\langle x\rangle)$, and

$$
\begin{aligned}
& \alpha_{\mathfrak{m}}(\langle x\rangle)=\frac{1}{m+2} \Delta_{\mathfrak{m}+1}+\sum_{\substack{n=-\infty \\
n \neq 0}}^{\infty}\left(-\frac{1}{m+2} \sum_{j=1}^{m} \frac{(m+2)_{j}}{(2 \pi i n)^{j}} \Delta_{m-j+1}\right) e^{2 \pi i n x} \\
& =\frac{1}{m+2} \Delta_{m+1}+\frac{1}{m+2} \sum_{j=1}^{m}\left(\begin{array}{c}
m+2 \\
j
\end{array}\right) \Delta_{m-j+1}\left(-j ! \sum_{\substack{n=-\infty \\
n \neq 0}}^{\infty} \frac{e^{2 \pi i n x}}{(2 \pi i n)^{j}}\right) \\
& =\frac{1}{m+2} \Delta_{m+1}+\frac{1}{m+2} \sum_{j=2}^{m}\left(\begin{array}{c}
m+2 \\
j
\end{array}\right) \Delta_{m-j+1} B_{j}(\langle x\rangle) \\
& +\Delta_{\mathrm{m}} \times \begin{cases}\mathrm{B}_{1}(\langle x\rangle), & \text { for } x \notin \mathbb{Z}, \\
0, & \text { for } x \in \mathbb{Z} .\end{cases}
\end{aligned}
$$

Now, we can state our first result.

Theorem 2.1. For each positive integer $l$, we put

$$
\Delta_{\mathrm{l}}=\sum_{\mathrm{k}=0}^{\mathrm{l}-1}\left(\mathrm{~B}_{\mathrm{k}}^{(\mathrm{r})}+(\mathrm{k}+1) \mathrm{B}_{\mathrm{k}}^{(\mathrm{r}-1)}\right) .
$$

Assume that $\Delta_{\mathrm{m}}=0$, for a positive integer $\mathrm{m}$. Then we have the following. 
(a) $\sum_{k=0}^{m} B_{k}^{(r)}(\langle x\rangle)\langle x\rangle^{m-k}$ has the Fourier series expansion

$$
\sum_{k=0}^{m} B_{k}^{(r)}(\langle x\rangle)\langle x\rangle^{m-k}=\frac{1}{m+2} \Delta_{m+1}+\sum_{\substack{n=-\infty \\ n \neq 0}}^{\infty}\left(-\frac{1}{m+2} \sum_{j=1}^{m} \frac{(m+2)_{j}}{(2 \pi i n)^{j}} \Delta_{m-j+1}\right) e^{2 \pi i n x},
$$

for all $x \in \mathbb{R}$, where the convergence is uniform.

(b)

$$
\sum_{k=0}^{m} B_{k}^{(r)}(\langle x\rangle)\langle x\rangle^{m-k}=\frac{1}{m+2} \Delta_{m+1}+\frac{1}{m+2} \sum_{j=2}^{m}\left(\begin{array}{c}
m+2 \\
j
\end{array}\right) \Delta_{m-j+1} B_{j}(\langle x\rangle),
$$

for all $x \in \mathbb{R}$, where $\mathrm{B}_{\mathbf{j}}(\langle x\rangle)$ is the Bernoulli function.

Assume next that $\Delta_{m} \neq 0$, for a positive integer $m$. Then $\alpha_{m}(0) \neq \alpha_{m}(1)$. Hence $\alpha_{m}(\langle x\rangle)$ is piecewise $\mathrm{C}^{\infty}$, and discontinuous with jump discontinuities at integers. The Fourier series of $\alpha_{\mathrm{m}}(\langle x\rangle)$ converges pointwise to $\alpha_{m}(\langle x\rangle)$, for $x \notin \mathbb{Z}$, and converges to

$$
\frac{1}{2}\left(\alpha_{m}(0)+\alpha_{m}(1)\right)=\alpha_{m}(0)+\frac{1}{2} \Delta_{m}
$$

for $x \in \mathbb{Z}$.

We can now state our second result.

Theorem 2.2. For each positive integer $l$, we put

$$
\Delta_{\mathrm{l}}=\sum_{\mathrm{k}=0}^{\mathrm{l}-1}\left(\mathrm{~B}_{\mathrm{k}}^{(\mathrm{r})}+(\mathrm{k}+1) \mathrm{B}_{\mathrm{k}}^{(\mathrm{r}-1)}\right) .
$$

Assume that $\Delta_{\mathrm{m}} \neq 0$, for a positive integer $\mathrm{m}$. Then we have the following.

(a)

$$
\begin{aligned}
& \frac{1}{\mathrm{~m}+2} \Delta_{\mathrm{m}+1}+\sum_{\substack{n=-\infty \\
n \neq 0}}^{\infty}\left(-\frac{1}{\mathrm{~m}+2} \sum_{j=1}^{\mathrm{m}} \frac{(\mathrm{m}+2)_{j}}{(2 \pi i n)^{j}} \Delta_{m-j+1}\right) e^{2 \pi i n x} \\
& \quad= \begin{cases}\sum_{k=0}^{m} B_{k}^{(r)}(\langle x\rangle)\langle x\rangle^{m-k}, & \text { for } x \notin \mathbb{Z}, \\
B_{m}^{(r)}+\frac{1}{2} \Delta_{m}, & \text { for } x \in \mathbb{Z} .\end{cases}
\end{aligned}
$$

(b)

$$
\begin{aligned}
& \frac{1}{m+2} \sum_{j=0}^{m}\left(\begin{array}{c}
m+2 \\
j
\end{array}\right) \Delta_{m-j+1} B_{j}(\langle x\rangle)=\sum_{k=0}^{m} B_{k}^{(r)}(\langle x\rangle)\langle x\rangle^{m-k}, \quad \text { for } x \notin \mathbb{Z}, \\
& \frac{1}{m+2} \sum_{\substack{j=0 \\
j \neq 1}}^{m}\left(\begin{array}{c}
m+2 \\
j
\end{array}\right) \Delta_{m-j+1} B_{j}(\langle x\rangle)=B_{m}^{(r)}+\frac{1}{2} \Delta_{m}, \quad \text { for } x \in \mathbb{Z} .
\end{aligned}
$$

\section{Fourier series of functions of the second type}

$$
\beta_{m}(x)=\sum_{k=0}^{m} \frac{1}{k !(m-k) !} B_{k}^{(r)}(x) x^{m-k}, \quad(m \geqslant 1) .
$$


Then we will consider the function

$$
\beta_{m}(\langle x\rangle)=\sum_{k=0}^{m} \frac{1}{k !(m-k) !} B_{k}^{(r)}(\langle x\rangle)\langle x\rangle^{m-k},
$$

defined on $\mathbb{R}$, which is periodic with period 1 .

The Fourier series of $\beta_{m}(\langle x\rangle)$ is

$$
\sum_{n=-\infty}^{\infty} E_{n}^{(m)} e^{2 \pi i n x}
$$

where

$$
\begin{aligned}
E_{n}^{(m)} & =\int_{0}^{1} \beta_{m}(\langle x\rangle) e^{-2 \pi i n x} d x \\
& =\int_{0}^{1} \beta_{m}(x) e^{-2 \pi i n x} d x .
\end{aligned}
$$

To proceed further, we need to observe the following.

$$
\begin{aligned}
\beta_{m}^{\prime}(x) & =\sum_{k=0}^{m}\left\{\frac{k}{k !(m-k) !} B_{k-1}^{(r)}(x) x^{m-k}+\frac{m-k}{k !(m-k) !} B_{k}^{(r)}(x) x^{m-k-1}\right\} \\
& =\sum_{k=1}^{m} \frac{1}{(k-1) !(m-k) !} B_{k-1}^{(r)}(x) x^{m-k}+\sum_{k=0}^{m-1} \frac{1}{k !(m-k-1) !} B_{k}^{(r)}(x) x^{m-k-1} \\
& =\sum_{k=0}^{m-1} \frac{1}{k !(m-1-k) !} B_{k}^{(r-1)}(x) x^{m-1-k}+\sum_{k=0}^{m-1} \frac{1}{k !(m-1-k) !} B_{k}^{(r)}(x) x^{m-1-k} \\
& =2 \beta_{m-1}(x) .
\end{aligned}
$$

From this, we have

$$
\left(\frac{\beta_{\mathrm{m}+1}(x)}{2}\right)^{\prime}=\beta_{\mathrm{m}}(x)
$$

and

$$
\int_{0}^{1} \beta_{m}(x) d x=\frac{1}{2}\left(\beta_{m+1}(1)-\beta_{m+1}(0)\right) .
$$

For $m \geqslant 1$, we put

$$
\begin{aligned}
\Omega_{m} & =\beta_{m}(1)-\beta_{m}(0) \\
& =\sum_{k=0}^{m} \frac{1}{k !(m-k) !}\left(B_{k}^{(r)}(1)-B_{k}^{(r)} \delta_{m, k}\right) \\
& =\sum_{k=0}^{m} \frac{1}{k !(m-k) !}\left(B_{k}^{(r)}+k B_{k-1}^{(r-1)}-B_{k}^{(r)} \delta_{m, k}\right) \\
& =\sum_{k=0}^{m} \frac{1}{k !(m-k) !} B_{k}^{(r)}+\sum_{k=1}^{m} \frac{1}{(k-1) !(m-k) !} B_{k-1}^{(r-1)}-\frac{1}{m !} B_{m}^{(r)} \\
& =\sum_{k=0}^{m-1} \frac{1}{k !(m-k) !} B_{k}^{(r)}+\sum_{k=0}^{m-1} \frac{1}{k !(m-k-1) !} B_{k}^{(r-1)} \\
& =\sum_{k=0}^{m-1} \frac{1}{k !(m-k) !}\left(B_{k}^{(r)}+(m-k) B_{k}^{(r-1)}\right) .
\end{aligned}
$$


From this, we see that

$$
\beta_{\mathrm{m}}(0)=\beta_{\mathrm{m}}(1) \Longleftrightarrow \Omega_{\mathrm{m}}=0,
$$

and

$$
\int_{0}^{1} \beta_{\mathrm{m}}(x) \mathrm{d} x=\frac{1}{2} \Omega_{\mathrm{m}+1}
$$

Now, we would like to determine the Fourier coefficients $E_{n}^{(m)}$.

Case $1: n \neq 0$.

$$
\begin{aligned}
E_{n}^{(m)} & =\int_{0}^{1} \beta_{m}(x) e^{-2 \pi i n x} d x \\
& =-\frac{1}{2 \pi i n}\left[\beta_{m}(x) e^{-2 \pi i n x}\right]_{0}^{1}+\frac{1}{2 \pi i n} \int_{0}^{1} \beta_{m}^{\prime}(x) e^{-2 \pi i n x} d x \\
& =-\frac{1}{2 \pi i n}\left(\beta_{m}(1)-\beta_{m}(0)\right)+\frac{2}{2 \pi i n x} \int_{0}^{1} \beta_{m-1}(x) e^{-2 \pi i n x} d x \\
& =\frac{2}{2 \pi i n} E_{n}^{(m-1)}-\frac{1}{2 \pi i n} \Omega_{m},
\end{aligned}
$$

from which by induction on $m$, we can deduce

$$
E_{n}^{(m)}=-\sum_{j=1}^{m} \frac{2^{j-1}}{(2 \pi i n)^{j}} \Omega_{m-j+1} .
$$

Case $2: n=0$.

$$
E_{0}^{(m)}=\int_{0}^{1} \beta_{m}(x) d x=\frac{1}{2} \Omega_{m+1} .
$$

$\beta_{m}(\langle x\rangle),(m \geqslant 1)$ is piecewise $C^{\infty}$. Moreover, $\beta_{m}(\langle x\rangle)$ is continuous for those positive integers $m$ with $\Omega_{\mathrm{m}}=0$ and discontinuous with jump discontinuities at integers for those positive integers $\mathrm{m}$ with $\Omega_{\mathrm{m}} \neq 0$.

Assume first that $\Omega_{m}=0$, for a positive integer $m$. Then $\beta_{m}(0)=\beta_{m}(1)$. Hence $\beta_{m}(\langle x\rangle)$ is piecewise $C^{\infty}$, and continuous. Thus the Fourier series of $\beta_{m}(\langle x\rangle)$ converges uniformly to $\beta_{m}(\langle x\rangle)$, and

$$
\begin{aligned}
\beta_{m}(\langle x\rangle)= & \frac{1}{2} \Omega_{m+1}+\sum_{\substack{n=-\infty \\
n \neq 0}}^{\infty}\left(-\sum_{j=1}^{m} \frac{2^{j-1}}{(2 \pi i n)^{j}} \Omega_{m-j+1}\right) e^{2 \pi i n x} \\
= & \frac{1}{2} \Omega_{m+1}+\sum_{j=1}^{m} \frac{2^{j-1}}{j !} \Omega_{m-j+1}\left(-j ! \sum_{\substack{n=-\infty \\
n \neq 0}}^{\infty} \frac{e^{2 \pi i n x}}{(2 \pi i n)^{j}}\right) \\
= & \frac{1}{2} \Omega_{m+1}+\sum_{\substack{j=2 \\
m}}^{\frac{2^{j-1}}{j !}} \Omega_{m-j+1} B_{j}(\langle x\rangle) \\
& +\Omega_{m} \times \begin{cases}B_{1}(\langle x\rangle), & \text { for } x \notin \mathbb{Z}, \\
0, & \text { for } x \in \mathbb{Z} .\end{cases}
\end{aligned}
$$

Now, we are ready to state the first result.

Theorem 3.1. For each positive integer $l$, we let

$$
\Omega_{l}=\sum_{k=0}^{l-1} \frac{1}{k !(l-k) !}\left(B_{k}^{(r)}+(l-k) B_{k}^{(r-1)}\right) .
$$

Assume that $\Omega_{\mathrm{m}}=0$, for a positive integer $\mathrm{m}$. Then we have the following. 
(a) $\sum_{k=0}^{m} \frac{1}{k !(m-k) !} B_{k}^{(r)}(\langle x\rangle)\langle x\rangle^{m-k}$ has the Fourier series expansion

$$
\sum_{k=0}^{m} \frac{1}{k !(m-k) !} B_{k}^{(r)}(\langle x\rangle)\langle x\rangle^{m-k}=\frac{1}{2} \Omega_{m+1}+\sum_{\substack{n=-\infty \\ n \neq 0}}^{\infty}\left(-\sum_{j=1}^{m} \frac{2^{j-1}}{(2 \pi i n)^{j}} \Omega_{m-j+1}\right) e^{2 \pi i n x},
$$

for all $x \in \mathbb{R}$, where the convergence is uniform.

(b)

$$
\sum_{k=0}^{m} \frac{1}{k !(m-k) !} B_{k}^{(r)}(\langle x\rangle)\langle x\rangle^{m-k}=\sum_{\substack{j=0 \\ j \neq 1}}^{m} \frac{2^{j-1}}{j !} \Omega_{m-j+1} B_{j}(\langle x\rangle),
$$

for all $x \in \mathbb{R}$, where $\mathrm{B}_{\mathbf{j}}(\langle x\rangle)$ is Bernoulli function.

Assume next that $\Omega_{m} \neq 0$, for a positive integer $m$. Then $\beta_{m}(0) \neq \beta_{m}(1)$. Hence $\beta_{m}(\langle x\rangle)$ is piecewise $C^{\infty}$, and discontinuous with jump discontinuities at integers. Thus the Fourier series of $\beta_{m}(\langle x\rangle)$ converges pointwise to $\beta_{\mathrm{m}}(\langle x\rangle)$, for $x \notin \mathbb{Z}$, and converges to

$$
\frac{1}{2}\left(\beta_{m}(0)+\beta_{m}(1)\right)=\beta_{m}(0)+\frac{1}{2} \Omega_{m},
$$

for $x \in \mathbb{Z}$.

Next, we can state our second result.

Theorem 3.2. For each positive integer $l$, we let

$$
\Omega_{l}=\sum_{k=0}^{l-1} \frac{1}{k !(l-k) !}\left(B_{k}^{(r)}+(l-k) B_{k}^{(r-1)}\right) .
$$

Assume that $\Omega_{\mathrm{m}} \neq 0$, for a positive integer $\mathrm{m}$. Then we have the following.

(a)

$$
\begin{aligned}
& \frac{1}{2} \Omega_{m+1}+\sum_{\substack{n=-\infty \\
n \neq 0}}^{\infty}\left(-\sum_{j=1}^{m} \frac{2^{j-1}}{(2 \pi i n)^{j}} \Omega_{m-j+1}\right) e^{2 \pi i n x} \\
& \quad= \begin{cases}\sum_{k=0}^{m} \frac{1}{k !(m-k) !} B_{k}^{(r)}(\langle x\rangle)\langle x\rangle^{m-k}, & \text { for } x \notin \mathbb{Z}, \\
\frac{1}{m !} B_{m}^{(r)}+\frac{1}{2} \Omega_{m}, & \text { for } x \in \mathbb{Z} .\end{cases}
\end{aligned}
$$

(b)

$$
\begin{aligned}
& \sum_{j=0}^{m} \frac{2^{j-1}}{j !} \Omega_{m-j+1} B_{j}(\langle x\rangle)=\sum_{k=0}^{m} \frac{1}{k !(m-k) !} B_{k}^{(r)}(\langle x\rangle)\langle x\rangle^{m-k}, \quad \text { for } x \notin \mathbb{Z}, \\
& \sum_{\substack{j=0 \\
j \neq 1}}^{m} \frac{2^{j-1}}{j !} \Omega_{m-j+1} B_{j}(\langle x\rangle)=\frac{1}{m !} B_{m}^{(r)}+\frac{1}{2} \Omega_{m}, \quad \text { for } x \in \mathbb{Z} .
\end{aligned}
$$

\section{Fourier series of functions of the third type}

Let

$$
\gamma_{m}(x)=\sum_{k=1}^{m-1} \frac{1}{k(m-k)} B_{k}^{(r)}(x) x^{m-k}, \quad(m \geqslant 2),
$$




$$
\begin{aligned}
\gamma_{m}^{\prime}(x) & =\sum_{k=1}^{m-1} \frac{1}{k(m-k)}\left\{k B_{k-1}^{(r)}(x) x^{m-k}+(m-k) B_{k}^{(r)}(x) x^{m-k-1}\right\} \\
& =\sum_{k=0}^{m-2} \frac{1}{m-1-k} B_{k}^{(r)}(x) x^{m-1-k}+\sum_{k=1}^{m-1} \frac{1}{k} B_{k}^{(r)}(x) x^{m-1-k} \\
& =\sum_{k=1}^{m-2}\left(\frac{1}{m-1-k}+\frac{1}{k}\right) B_{k}^{(r)}(x) x^{m-1-k}+\frac{1}{m-1} x^{m-1}+\frac{1}{m-1} B_{m-1}^{(r)}(x) \\
& =(m-1) \sum_{k=1}^{m-2} \frac{1}{k(m-1-k)} B_{k}^{(r)}(x) x^{m-1-k}+\frac{1}{m-1} x^{m-1}+\frac{1}{m-1} B_{m-1}^{(r)}(x) \\
& =(m-1) \gamma_{m-1}(x)+\frac{1}{m-1} x^{m-1}+\frac{1}{m-1} B_{m-1}^{(r)}(x) .
\end{aligned}
$$

Thus

$$
\gamma_{m}^{\prime}(x)=(m-1) \gamma_{m-1}(x)+\frac{1}{m-1} x^{m-1}+\frac{1}{m-1} B_{m-1}^{(r)}(x),
$$

from which we see that

$$
\left(\frac{1}{m}\left(\gamma_{m+1}(x)-\frac{1}{m(m+1)} x^{m+1}-\frac{1}{m(m+1)} B_{m+1}^{(r)}(x)\right)\right)^{\prime}=\gamma_{m}(x) .
$$

This implies that

$$
\begin{aligned}
\int_{0}^{1} \gamma_{m}(x) d x & =\frac{1}{m}\left(\gamma_{m+1}(1)-\gamma_{m+1}(0)-\frac{1}{m(m+1)}-\frac{1}{m(m+1)}\left(B_{m+1}^{(r)}(1)-B_{m+1}^{(r)}\right)\right) \\
& =\frac{1}{m}\left(\gamma_{m+1}(1)-\gamma_{m+1}(0)-\frac{1}{m(m+1)}-\frac{1}{m} B_{m}^{(r-1)}\right) .
\end{aligned}
$$

For $m \geqslant 2$, we put

$$
\begin{aligned}
\Lambda_{m}=\gamma_{m}(1)-\gamma_{m}(0) & =\sum_{k=1}^{m-1} \frac{1}{k(m-k)}\left(B_{k}^{(r)}(1)-B_{k}^{(r)} \delta_{m, k}\right) \\
& =\sum_{k=1}^{m-1} \frac{1}{k(m-k)}\left(B_{k}^{(r)}+k B_{k-1}^{(r-1)}-B_{k}^{(r)} \delta_{m, k}\right) \\
& =\sum_{k=1}^{m-1} \frac{1}{k(m-k)}\left(B_{k}^{(r)}+k B_{k-1}^{(r-1)}\right) .
\end{aligned}
$$

Now, we note that

$$
\gamma_{\mathrm{m}}(0)=\gamma_{\mathrm{m}}(1) \Longleftrightarrow \Lambda_{\mathrm{m}}=0,
$$

and

We now consider the function

$$
\int_{0}^{1} \gamma_{m}(x) d x=\frac{1}{m}\left(\Lambda_{m+1}-\frac{1}{m(m+1)}-\frac{1}{m} B_{m}^{(r-1)}\right) .
$$

$$
\gamma_{m}(\langle x\rangle)=\sum_{k=1}^{m-1} \frac{1}{k(m-k)} B_{k}^{(r)}(\langle x\rangle)\langle x\rangle^{m-k}, \quad(m \geqslant 2),
$$

defined on $\mathbb{R}$, which is periodic with period 1 . The Fourier series of $\gamma_{m}(\langle x\rangle)$ is

$$
\sum_{n=-\infty}^{\infty} C_{n}^{(m)} e^{2 \pi i n x}
$$


where

$$
\begin{aligned}
C_{n}^{(m)} & =\int_{0}^{1} \gamma_{m}(\langle x\rangle) e^{-2 \pi i n x} d x \\
& =\int_{0}^{1} \gamma_{m}(x) e^{-2 \pi i n x} d x
\end{aligned}
$$

We are now going to determine the Fourier coefficients $C_{n}^{(m)}$.

Case $1: n \neq 0$. We can show that

$$
\int_{0}^{1} x^{l} e^{-2 \pi i n x} d x= \begin{cases}-\sum_{k=1}^{l} \frac{(l)_{k-1}}{(2 \pi i n)^{k}}, & \text { for } n \neq 0 \\ \frac{1}{l+1}, & \text { for } n=0\end{cases}
$$

Also, from [13] we have

$$
\begin{aligned}
& \int_{0}^{1} B_{l}^{(r)}(x) e^{-2 \pi i n x} d x= \begin{cases}-\sum_{k=1}^{l} \frac{(l)_{k}}{(2 \pi i n)^{k}} B_{l-k}^{(r-1)}, & \text { for } n \neq 0, \\
B_{l}^{(r-1)}, & \text { for } n=0 .\end{cases} \\
& C_{n}^{(m)}=\int_{0}^{1} \gamma_{m}(x) e^{-2 \pi i n x} d x \\
&=-\frac{1}{2 \pi i n}\left[\gamma_{m}(x) e^{-2 \pi i n x}\right]_{0}^{1}+\frac{1}{2 \pi i n} \int_{0}^{1} \gamma_{m}^{\prime}(x) e^{-2 \pi i n x} d x \\
&=-\frac{1}{2 \pi i n}\left(\gamma_{m}(1)-\gamma_{m}(0)\right) \\
&+\frac{1}{2 \pi i n} \int_{0}^{1}\left((m-1) \gamma_{m-1}(x)+\frac{1}{m-1} x^{m-1}+\frac{1}{m-1} B_{m-1}^{(r)}(x)\right) e^{-2 \pi i n x} d x \\
&= \frac{m-1}{2 \pi i n} C_{n}^{(m-1)}-\frac{1}{2 \pi i n} \Lambda_{m}-\frac{1}{2 \pi i n(m-1)} \Phi_{m}-\frac{1}{2 \pi i n(m-1)} \Theta_{m},
\end{aligned}
$$

where

$$
\Phi_{m}=\sum_{k=1}^{m-1} \frac{(m-1)_{k-1}}{(2 \pi i n)^{k}}, \quad \Theta_{m}=\sum_{k=1}^{m-1} \frac{(m-1)_{k}}{(2 \pi i n)^{k}} B_{m-k-1}^{(r-1)} .
$$

Thus we have shown that

$$
C_{n}^{(m)}=\frac{m-1}{2 \pi i n} C_{n}^{(m-1)}-\frac{1}{2 \pi i n} \Lambda_{m}-\frac{1}{2 \pi i n(m-1)} \Phi_{m}-\frac{1}{2 \pi i n(m-1)} \Theta_{m},
$$

from which by induction on $m$ we can show that

$$
\begin{aligned}
C_{n}^{(m)}= & -\sum_{j=1}^{m-1} \frac{(m-1)_{j-1}}{(2 \pi i n)^{j}} \Lambda_{m-j+1}-\sum_{j=1}^{m-1} \frac{(m-1)_{j-1}}{(2 \pi i n)^{j}(m-j)} \Phi_{m-j+1} \\
& -\sum_{j=1}^{m-1} \frac{(m-1)_{j-1}}{(2 \pi i n)^{j}(m-j)} \Theta_{m-j-1} .
\end{aligned}
$$

We observe now that

$$
\sum_{j=1}^{m-1} \frac{(m-1)_{j-1}}{(2 \pi i n)^{j}(m-j)} \Theta_{m-j+1}=\sum_{j=1}^{m-1} \frac{(m-1)_{j-1}}{(2 \pi i n)^{j}(m-j)} \sum_{k=1}^{m-j} \frac{(m-j)_{k}}{(2 \pi i n)^{k}} B_{m-j-k}^{(r-1)}
$$




$$
\begin{aligned}
& =\sum_{j=1}^{m-1} \frac{1}{m-j} \sum_{k=1}^{m-j} \frac{(m-1)_{j+k-1}}{(2 \pi i n)^{j+k}} B_{m-j-k}^{(r-1)} \\
& =\sum_{j=1}^{m-1} \frac{1}{m-j} \sum_{s=j+1}^{m} \frac{(m-1)_{s-1}}{(2 \pi i n)^{s}} B_{m-s}^{(r-1)} \\
& =\sum_{s=2}^{m} \frac{(m-1)_{s-1}}{(2 \pi i n)^{s}} B_{m-s}^{(r-1)} \sum_{j=1}^{s-1} \frac{1}{m-j} \\
& =\frac{1}{m} \sum_{s=1}^{m} \frac{(m)_{s}}{(2 \pi i n)^{s}} B_{m-s}^{(r-1)}\left(H_{m-1}-H_{m-s}\right),
\end{aligned}
$$

where $H_{m}=\sum_{j=1}^{m} \frac{1}{j}$ are harmonic numbers. Similarly, we can show that

$$
\sum_{j=1}^{m-1} \frac{(m-1)_{j-1}}{(2 \pi i n)^{j}(m-j)} \Phi_{m-j+1}=\frac{1}{m} \sum_{s=1}^{m} \frac{(m)_{s}}{(2 \pi i n)^{s}} \frac{H_{m-1}-H_{m-s}}{m-s+1} .
$$

Putting everything altogether, from (4.1) we have

$$
C_{n}^{(m)}=-\frac{1}{m} \sum_{s=1}^{m} \frac{(m)_{s}}{(2 \pi i n)^{s}}\left\{\Lambda_{m-s+1}+\left(H_{m-1}-H_{m-s}\right)\left(\frac{1}{m-s+1}+B_{m-s}^{(r-1)}\right)\right\} .
$$

Case $2: n=0$.

$$
\begin{aligned}
C_{0}^{(m)} & =\int_{0}^{1} \gamma_{m}(x) d x \\
& =\frac{1}{m}\left(\Lambda_{m+1}-\frac{1}{m(m+1)}-\frac{1}{m} B_{m}^{(r-1)}\right) .
\end{aligned}
$$

$\gamma_{m}(\langle x\rangle),(m \geqslant 2)$ is piecewise $C^{\infty}$. Moreover, $\gamma_{m}(\langle x\rangle)$ is continuous for those integers $m \geqslant 2$ with $\Lambda_{m}=0$ and discontinuous with jump discontinuities at integers for those integers $m \geqslant 2$ with $\Lambda_{m} \neq 0$.

Assume first that $\Lambda_{m}=0$, for an integer $m \geqslant 2$. Then $\gamma_{m}(0)=\gamma_{m}(1)$. Hence $\gamma_{m}(\langle x\rangle)$ is piecewise $\mathrm{C}^{\infty}$, and continuous. Thus the Fourier series of $\gamma_{\mathrm{m}}(\langle x\rangle)$ converges uniformly to $\gamma_{\mathrm{m}}(\langle x\rangle)$, and

$$
\begin{aligned}
& \gamma_{m}(\langle x\rangle)=\frac{1}{m}\left(\Lambda_{m+1}-\frac{1}{m(m+1)}-\frac{1}{m} B_{m}^{(r-1)}\right)-\sum_{\substack{n=-\infty \\
n \neq 0}}^{\infty}\left\{\frac { 1 } { m } \sum _ { s = 1 } ^ { m } \frac { ( m ) _ { s } } { ( 2 \pi i n ) ^ { s } } \left(\Lambda_{m-s+1}\right.\right. \\
& \left.\left.+\left(\mathrm{H}_{\mathrm{m}-1}-\mathrm{H}_{\mathrm{m}-\mathrm{s}}\right)\left(\frac{1}{\mathrm{~m}-\mathrm{s}+1}+\mathrm{B}_{\mathrm{m}-\mathrm{s}}^{(\mathrm{r}-1)}\right)\right)\right\} e^{2 \pi i n x} \\
& =\frac{1}{m}\left(\Lambda_{m+1}-\frac{1}{m(m+1)}-\frac{1}{m} B_{m}^{(r-1)}\right)+\frac{1}{m} \sum_{s=1}^{m}\left(\begin{array}{c}
m \\
s
\end{array}\right)\left(\Lambda_{m-s+1}\right. \\
& \left.+\left(H_{m-1}-H_{m-s}\right)\left(\frac{1}{m-s+1}+B_{m-s}^{(r-1)}\right)\right)\left(-s ! \sum_{\substack{n=-\infty \\
n \neq 0}}^{\infty} \frac{e^{2 \pi i n x}}{(2 \pi i n)^{s}}\right) \\
& =\frac{1}{m}\left(\Lambda_{m+1}-\frac{1}{m(m+1)}-\frac{1}{m} B_{m}^{(r-1)}\right)+\frac{1}{m} \sum_{s=2}^{m}\left(\begin{array}{c}
m \\
s
\end{array}\right)\left(\Lambda_{m-s+1}+\left(H_{m-1}-H_{m-s}\right)\right. \\
& \left.\times\left(\frac{1}{m-s+1}+B_{m-s}^{(r-1)}\right)\right) B_{s}(\langle x\rangle)+\Lambda_{m} \times \begin{cases}B_{1}(\langle x\rangle), & \text { for } x \notin \mathbb{Z}, \\
0, & \text { for } x \in \mathbb{Z} .\end{cases}
\end{aligned}
$$

Now, we are ready to state our first result. 
Theorem 4.1. For each integer $l \geqslant 2$, we let

$$
\Lambda_{l}=\sum_{k=1}^{l-1} \frac{1}{k(l-k)}\left(B_{k}^{(r)}+k B_{k-1}^{(r-1)}\right),
$$

with $\Lambda_{1}=0$. Assume that $\Lambda_{\mathrm{m}}=0$, for an integer $\mathrm{m} \geqslant 2$. Then we have the following.

(a) $\sum_{k=1}^{m-1} \frac{1}{k(m-k)} B_{k}^{(r)}(\langle x\rangle)\langle x\rangle^{m-k}$ has the Fourier series expansion

$$
\begin{aligned}
\sum_{k=1}^{m-1} & \frac{1}{k(m-k)} B_{k}^{(r)}(\langle x\rangle)\langle x\rangle^{m-k} \\
= & \frac{1}{m}\left(\Lambda_{m+1}-\frac{1}{m(m+1)}-\frac{1}{m} B_{m}^{(r-1)}\right)-\sum_{\substack{n=-\infty \\
n \neq 0}}^{\infty}\left\{\frac { 1 } { m } \sum _ { s = 1 } ^ { m } \frac { ( m ) _ { s } } { ( 2 \pi i n ) ^ { s } } \left(\Lambda_{m-s+1}\right.\right. \\
& \left.\left.+\left(H_{m-1}-H_{m-s}\right)\left(\frac{1}{m-s+1}+B_{m-s}^{(r-1)}\right)\right)\right\} e^{2 \pi i n x}
\end{aligned}
$$

for all $x \in \mathbb{R}$, where the convergence is uniform.

(b)

$$
\begin{aligned}
& \sum_{k=1}^{m-1} \frac{1}{k(m-k)} B_{k}^{(r)}(\langle x\rangle)\langle x\rangle^{m-k} \\
& \quad=\frac{1}{m} \sum_{\substack{s=0 \\
s \neq 1}}^{m}\left(\begin{array}{c}
m \\
s
\end{array}\right)\left(\Lambda_{m-s+1}+\left(H_{m-1}-H_{m-s}\right)\left(\frac{1}{m-s+1}+B_{m-s}^{(r-1)}\right)\right) B_{s}(\langle x\rangle),
\end{aligned}
$$

for all $x \in \mathbb{R}$, where $\mathrm{B}_{\mathrm{s}}(\langle x\rangle)$ is the Bernoulli function.

Assume next that $\Lambda_{m} \neq 0$, for an integer $m \geqslant 2$. Then $\gamma_{m}(0) \neq \gamma_{m}(1)$. Hence $\gamma_{m}(\langle x\rangle)$ is piecewise $C^{\infty}$, and discontinuous with jump discontinuities at integers. Thus the Fourier series of $\gamma_{m}(\langle x\rangle)$ converges pointwise to $\gamma_{m}(\langle x\rangle)$, for $x \notin \mathbb{Z}$ and converges to

$$
\begin{aligned}
\frac{1}{2}\left(\gamma_{\mathrm{m}}(0)+\gamma_{\mathrm{m}}(1)\right) & =\gamma_{\mathrm{m}}(0)+\frac{1}{2} \Lambda_{\mathrm{m}} \\
& =\frac{1}{2} \Lambda_{\mathrm{m}},
\end{aligned}
$$

for $x \in \mathbb{Z}$. We are ready to state our second result.

Theorem 4.2. For each integer $l \geqslant 2$, we let

$$
\Lambda_{l}=\sum_{k=1}^{l-1} \frac{1}{k(l-k)}\left(B_{k}^{(r)}+k B_{k-1}^{(r-1)}\right),
$$

with $\Lambda_{1}=0$. Assume that $\Lambda_{m} \neq 0$, for an integer $m \geqslant 2$. Then we have the following.

(a)

$$
\begin{gathered}
\frac{1}{m}\left(\Lambda_{m+1}-\frac{1}{m(m+1)}-\frac{1}{m} B_{m}^{(r-1)}\right)-\sum_{\substack{n=-\infty \\
n \neq 0}}^{\infty}\left\{\frac { 1 } { m } \sum _ { s = 1 } ^ { m } \frac { ( m ) _ { s } } { ( 2 \pi i n ) ^ { s } } \left(\Lambda_{m-s+1}\right.\right. \\
\left.\left.+\left(H_{m-1}-H_{m-s}\right)\left(\frac{1}{m-s+1}+B_{m-s}^{(r-1)}\right)\right)\right\} e^{2 \pi i n x} \\
= \begin{cases}\sum_{k=1}^{m-1} \frac{1}{k(m-k)} B_{k}^{(r)}(\langle x\rangle)\langle x\rangle^{m-k}, & \text { for } x \notin \mathbb{Z}, \\
\frac{1}{2} \Lambda_{m}, & \text { for } x \in \mathbb{Z} .\end{cases}
\end{gathered}
$$


(b)

$$
\begin{aligned}
& \frac{1}{m} \sum_{s=0}^{m}\left(\begin{array}{c}
m \\
s
\end{array}\right)\left(\Lambda_{m-s+1}+\left(H_{m-1}-H_{m-s}\right)\left(\frac{1}{m-s+1}+B_{m-s}^{(r-1)}\right)\right) B_{s}(\langle x\rangle) \\
& \quad=\sum_{k=1}^{m-1} \frac{1}{k(m-k)} B_{k}^{(r)}(\langle x\rangle)\langle x\rangle^{m-k}, \quad \text { for } x \notin \mathbb{Z} ; \\
& \frac{1}{m} \sum_{\substack{s=0 \\
s \neq 1}}^{m}\left(\begin{array}{c}
m \\
s
\end{array}\right)\left(\Lambda_{m-s+1}+\left(H_{m-1}-H_{m-s}\right)\left(\frac{1}{m-s+1}+B_{m-s}^{(r-1)}\right)\right) B_{s}(\langle x\rangle) \\
& \quad=\frac{1}{2} \Lambda_{m}, \quad \text { for } x \in \mathbb{Z} .
\end{aligned}
$$

\section{Acknowledgment}

This research was supported by the Daegu University Research Grant, 2016.

\section{References}

[1] M. Abramowitz, I. A. Stegun, Handbook of mathematical functions with formulas, graphs, and mathematical tables, National Bureau of Standards Applied Mathematics Series, For sale by the Superintendent of Documents, U.S. Government Printing Office, Washington, D.C., (1964). 1

[2] L. Carlitz, A note on Bernoulli numbers and polynomials, Elem. Math., 29 (1974), 90-92. 1.1

[3] G. V. Dunne, C. Schubert, Bernoulli number identities from quantum field theory and topological string theory, Commun. Number Theory Phys., 7 (2013), 225-249. 1

[4] C. Faber, R. Pandharipande, Hodge integrals and Gromov-Witten theory, Invent. Math., 139 (2000), 173-199. 1

[5] I. M. Gessel, On Miki's identity for Bernoulli numbers, J. Number Theory, 110 (2005), 75-82. 1

[6] H. W. Gould, Explicit formulas for Bernoulli numbers, Amer. Math. Monthly, 79 (1972), 44-51. 1.1

[7] D. S. Kim, T. Kim, Bernoulli basis and the product of several Bernoulli polynomials, Int. J. Math. Math. Sci., 2012 (2012), 12 pages. 1

[8] D. S. Kim, T. Kim, A note on higher-order Bernoulli polynomials, J. Inequal. Appl., 2013 (2013), 9 pages. 1.1

[9] D. S. Kim, T. Kim, Some identities of higher order Euler polynomials arising from Euler basis, Integral Transforms Spec. Funct., 24 (2013), 734-738. 1

[10] D. S. Kim, T. Kim, Higher-order Bernoulli and poly-Bernoulli mixed type polynomials, Georgian Math. J., 22 (2015), 265-272. 1.1

[11] D. S. Kim, T. Kim, Fourier series of higher-order Euler functions and their applications, Bull. Korean Math. Soc., (to appear). 1

[12] D. S. Kim, T. Kim, Y. H. Kim, S.-H. Lee, Some arithmetic properties of Bernoulli and Euler numbers, Adv. Stud. Contemp. Math. (Kyungshang), 22 (2012), 467-480. 1.1

[13] T. Kim, D. S. Kim, S.-H. Rim, D. V. Dolgy, Fourier series of higher-order Bernoulli functions and their applications, J. Inequal. Appl., 2017 (2017), 7 pages. 1, 4

[14] D. H. Lehmer, A new approach to Bernoulli polynomials, Amer. Math. Monthly, 95 (1988), 905-911. 1.1

[15] J. E. Marsden, Elementary classical analysis, With the assistance of Michael Buchner, Amy Erickson, Adam Hausknecht, Dennis Heifetz, Janet Macrae and William Wilson, and with contributions by Paul Chernoff, István Fáry and Robert Gulliver, W. H. Freeman and Co., San Francisco, (1974). 1

[16] K. Shiratani, S. Yokoyama, An application of p-adic convolutions, Mem. Fac. Sci. Kyushu Univ. Ser. A, 36 (1982), 73-83. 1

[17] L. C. Washington, Introduction to cyclotomic fields, Second edition, Graduate Texts in Mathematics, Springer-Verlag, New York, (1997). 1.1

[18] D. G. Zill, M. R. Cullen, Advanced engineering mathematics, Second edition, Jones and Bartlett Publishers, London, UK, (2000). 1 